\title{
Solar Radius Variations Measured in Central Eclipses
}

\author{
Costantino Sigismondi \\ Sapienza Università di Roma and ICRA, International Center for Relativistic Astrophysics
Piazzale Aldo Moro 5,00185 Roma Italia email: sigismondi@icra.it
}

\begin{abstract}
Data on Baily beads observed in total eclipse of March 29, 2006 (Egypt) and those of annular eclipses of September 22, 2006 (French Guyana) and October 3, 2005 (Spain) are used to evaluate the variations of solar radius with respect to its standard value during a whole draconitic year. A portable observatory has to be set on the shadow limit of central eclipses, where lunar limb is grazing to the solar one and the number of beads is large. The observation of solar corona during Egyptian eclipse for several minutes during maximum eclipse on shadow's limits is studied in parallel with the eclipse observed by Clavius in 1567. From fall 2005 to fall 2006 the solar radius does not show significant changes ( 0.00 to -0.01 arcsecs) with respect to its standard value of 959.63 arcsec within errorbars of 0.17 arcsecs. This is its value at minimum of cycle 23 of solar activity.
\end{abstract}

Keywords: Eclispes; Ephemerides; Astrometry; Solar Physics; Diameter; Photosphere; Solar Variability. PACS: 95.10.Gi; 95.10.Km; 95.10.Jk; 96.60.-j; 96.60.Bn; 96.60.Mz; 92.70.Qr.

\section{INTRODUCTION}

Solar eclipses have been studied since antiquity and their predictions have been compared with observations to check solar and lunar theories used in the ephemerides. The case of the eclipse of June 21, 1629 (partial on Beijing) is famous: two Jesuits G. Schreck and G. A. Schall von Bell predicted its circumstances better than Chinese astronomers and after that they were charged to set a calendar reformation (Maffei, 1984). Recently ancient eclipse data contributed to track back to VIII century B.C. the Earth spin rate and to recover the lunar secular acceleration (Stephenson, 1997). Finally simulations on April 9, 1567 eclipse(Eddy and Boornazian, 1979 and Stephenson, Jones and Morrison, 1997), that Clavius (1581) observed as annular, opened the astrophysical question about secular solar radius variations (Parkinson et al. 1988; Thuillier, Haberreiter and Sofia, 2005).

\section{HISTORICAL ECLIPSES}

In 1715 Edmund Halley steered the observational campaign of Royal Society all across Southern England, to find the umbral limits of May $3^{\text {rd }}$ total eclipse passing over London (Halley, 1717).

For 1925, January $24^{\text {th }}$ eclipse Ernest W. Brown (1926), professor at Yale University, encouraged a large scale observational campaign. He recovered the Southern umbral limit over Manhattan with one block ( 100 m) of accuracy thanks to the account of some employees of an electric company who wanted to study the correlation between environmental illumination and consumes. They were placed on the roofs with one block spacing. The Northern limit was individuated near Providence (Rhode Island).

\section{The Marginal Zones of the Moon}

In 1963 Chester Watts at US Naval Observatory completed the accurate profiles of the atlas of the Marginal Zones of the Moon, whose valleys determined Baily Beads features when they are on the line of sight of solar limb. The atlas has been computed in more than 18 years for all lunar libration phases. That atlas was prepared for using lunar occultations to determine accurately the longitude of the observer, and it was the most accurate location's finding tool before GPS. After 60000 observations of lunar occultations Morrison and Appleby (1981) corrected Watts' charts for a systematic error, while the uncertainty for a single feature is $\sigma \leq 0.2 \operatorname{arcsec}$.

\section{The Saros Cycle and Lunar Libration Phases}

Saros cycle of eclipses is known since antiquity, it is 18 years and 10.3 (11.3) days. Eclipses occurs with $8 \mathrm{~h}\left(120^{\circ}\right.$ of longitude) of difference from previous one. The Moon is at the orbital nodes and in the same synodical phase after 228 synodic and 242 draconitic months. Moreover the Moon shows the same libration phase, and in the eclipse are produced the same Baily beads. Three Saros cycles is an Exeligmos, it lasts 54 years and 32 or 33 days, depending on leap years sequence, and the eclipse occurs in the same region and hour of 54 years before. 
On February 26, 1979 a total eclipse occurred again over United States, Saros-connected with to that one of 1925 and it has been observed for measuring variations from the previous ones (Dunham et al., 1980). Comparing Saros-connected eclipses allow treat as systematic errors that ones on Watts charts.

Furthermore during an eclipse libration phase in latitude is nearly zero, so the polar Baily beads are produced by the same limb features. This encouraged D. Dunham (1973 and 2005) to observe solar grazes, where the lunar limb trajectory is tangent to the solar one: there polar beads are the last ones to disappear, or they remain to shine like a single bright star for some seconds.

\section{Secular Variations of Solar Radius}

Analyzing Clavius' observation of 1567 April $9^{\text {th }}$ annular eclipse in Rome claiming Eddy and Boornazian (1979) claimed a larger Sun ( 2.5 arcsec) for that epoch while Morrison et al. (1997) rejected this hypothesis. Dunham et al. (1980) started to observe grazing eclipses and to gather historical data in order to verify sub-arcsecond variations of solar radius. Eclipse data have been gathered and analyzed by Alan Fiala, David Dunham, Sabatino Sofia (Fiala et al. 1994) on the purpose of measuring solar radius variations in the last three centuries.

TABLE 1. Corrections to average solar radius (959.63 arcsec) from Fiala et al. (1994)+our data

\begin{tabular}{ccc}
\hline Date & $\begin{array}{c}\text { Type of } \\
\text { Eclipse }\end{array}$ & $\begin{array}{c}\text { Correction to solar } \\
\text { radius }\end{array}$ \\
\hline May 3 1715 & Total & $\Delta R=(+0.48 \pm 0.02)$ \\
Jan 24 1925 & Total & $\Delta R=(+0.51 \pm 0.08)$ \\
Oct 23 1976 & Total & $\Delta R=(+0.04 \pm 0.07)$ \\
Feb 26 1979 & Total & $\Delta R=(-0.11 \pm 0.05)$ \\
Feb 16 1980 & Total & $\Delta R=(-0.03 \pm 0.03)$ \\
Feb 4 1981 & Annular & $\Delta R=(-0.02 \pm 0.03)$ \\
June 11 1983 & Total & $\Delta R=(+0.09 \pm 0.02)$ \\
May 30 1984 & Annular & $\Delta R=(+0.09 \pm 0.04)$ \\
Sep 23 1987 & Annular & $\Delta R=(-0.11 \pm 0.03)$ \\
Oct. 3 2005 & Annular & $\Delta R=(0.00 \pm 0.17)$ \\
Sept 22 2006 & Annular & $\Delta R=(-0.01 \pm 0.17)$ \\
\hline
\end{tabular}

David Herald (2001-2006) developed a computer program under DOS and Windows with digitized Watts' charts in and able to simulate all the eclipse's features and simplify the procedures of data reduction. This program has been used to plan the choice of observational points and to reduce the data of recorded Baily Beads.

Accurate studies on historical and recent data have been made also by Parkinson et al. (1988), Brown et al. (1998). Thuillier, Sofia and Haberreiter (2005) present an experimental evidence of anticorrelation between solar spots and solar diameter. Radius fluctuations range within 0.20 arcsec with an 11 year period.

From the astrophysical point of view several studies concerned the Sun and its variability: Sofia et al. (1979 and 1983); Endal et al. (1985) are the seminal works in this field.

The mission SDS, Solar Disk Sextant, was designed to overcome the atmospheric seeing using a balloon borne telescope at a quote of $37 \mathrm{Km}$, where the residual atmosphere has a pressure of $3 \mathrm{hPa}$. The accuracy of the solar radius corrections was within 20 milliarcseconds for (Egidi, Caccin et al., 2006). The solar radius has been measured in the bandwidth $590-670 \mathrm{~nm}$ during a series of flights carried out in 1992, 1994, 1995, and 1996. The measured radius value appears anti-correlated with the level of solar activity, ranging from about 959.5 to 959.7 arcsec.

PICARD satellite (Damé et al., 1999) will be launched in 2009 and it is expected to get a milliarcsecond accuracy in the solar radius variations.

\section{SOLAR GRAZES}

When a star grazes the lunar limb during an occultation that occultation is called graze. Those phenomena help astronomers to map accurately lunar polar regions profiles, especially in the Cassini regions, which are never illuminated because of lunar libration phases (Povenmire, 1975), and are lacking in Watts atlas. A solar graze is the same phenomenon at the limits of umbra or of annularity in total eclipses.

The formation of Baily Beads occurs when solar and lunar limbs are nearly tangent, and for grazes they can appear over several degrees of position angles, depending on relative dimensions of Moon and Sun's disks. The phenomenon can last several minutes, while at the eclipse's centerline it lasts only few seconds and it is limited to equatorial position angles.

At the shadow limit the solar corona is visible to the naked eye for a duration slightly larger than being on the centerline. It is possible to observe it covering the photospheric beads with the thumb as experienced in the Egyptian eclipse of March 29, 2006.

Illumination changes are slower at the limbs than on centerline as it is visible in the sequences of figure 1 and 2 .

The width of umbral band depends on the distance $d$ of the Moon from Earth surface (subsolar point) and $\delta$, the difference between lunar and solar diameter. $\mathrm{W}=\mathrm{d} \cdot \tan (\delta)$. In Egypt it was $\mathrm{d}=353900$ $\mathrm{Km}, \delta==2026-1922=104$ arcsec and $\mathrm{W}=178 \mathrm{Km}$. 

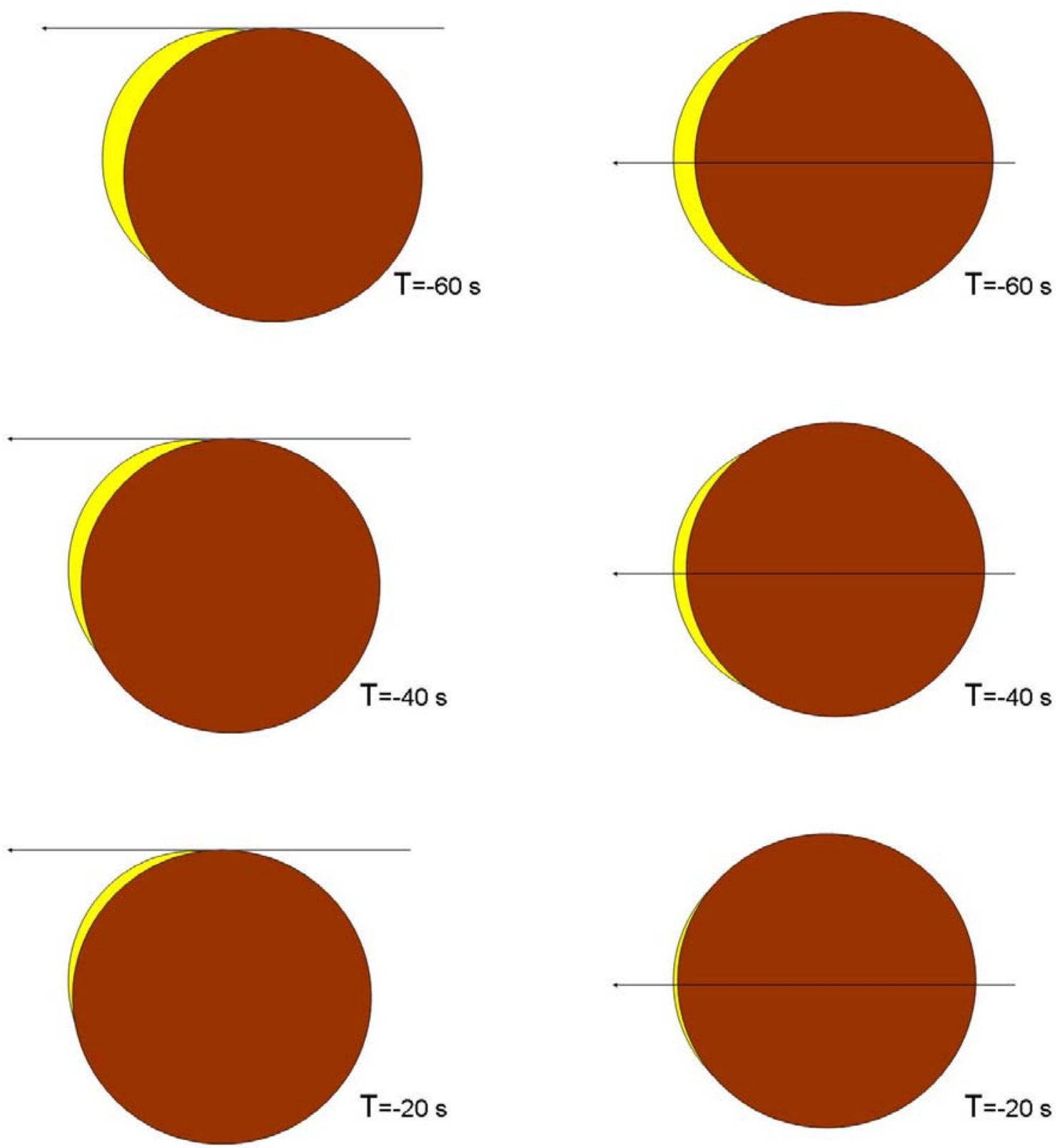

FIGURE 1. Sequence of total eclipse as seen from shadow's limit (left hand side) and from centerline (right hand side). The profile of the Moon is shifted of same steps of $20 \mathrm{~s}$, from top to bottom. The region where Baily Beads form is limited to equatorial zone in centerline (right) while it is extended from equator to poles in grazing eclipses.

A rotating crescent is observed during the maximum phases of the eclipse just outside the shadow's limit, while on the limit the angles of beads' formation rotate from West to East.

Acknowledgments: Thanks to my collaborating students during 2006 and 2007: Micol Benetti, Paolo Fermani, Marco Innocenti, Alessandra Mastrobuono Battisti, Chiara Melchiorre, Irene di Palma, Silvia Pietroni. Thanks to Paolo Colona and to Dr. Fady Morcos for Egyptian Eclipse. Thanks to Prof. Albert Picciocchi for Guyana eclipse. Thanks to Prof. Remo Ruffini for supporting those observational champaign. Thanks to Pietro Oliva, Paolo Colona, to the Sabadell Agrupaciò de Astronomia, and to Dr. Wolgang Strickling for Spanish eclipse data. 

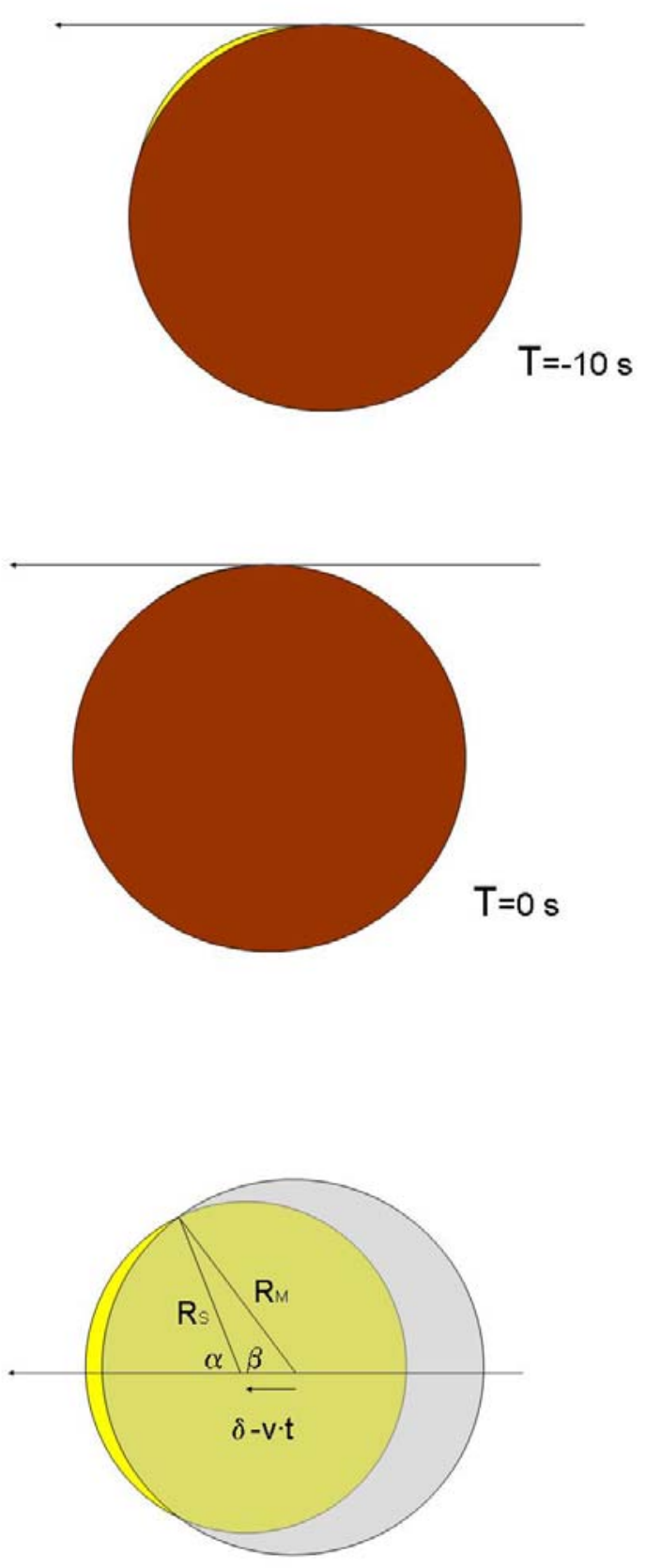

FIGURE 3. Geometry for calculation of visible sun area. Case of centerline. The Moon is moving at velocity $\mathrm{v}$ toward left (East, position angles around $90^{\circ}-28^{\circ}$ in March, 292006 and $90^{\circ}+28^{\circ}$ in September 22, 2006). At totality $\mathrm{t}=0$, while before totality $\mathrm{t}<0$, so the distance of the centers of Sun and Moon is larger than $\delta$, the difference of their radii. Rs stands for solar radius, $\mathrm{Rm}$ for Moon radius.

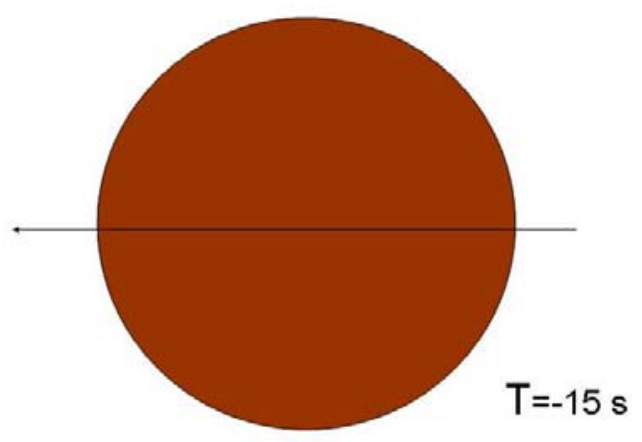

FIGURE 2. Totality in centerline (right hand side) and on shadow limits (left): following the previous figure the totality starts at $\mathrm{t}=-15 \mathrm{~s}$ on centerline, while in left hand sequence the total darkness occurs only at $\mathrm{t}=0$.

In order to calculate the variation of illumination in the two cases of centerline and shadow's limit we evaluate the Sun area A visible from geometric calculations. The role of Baily beads is neglected in this approach. The area A h·a is proportional to the height $\mathrm{h}$ of the Sun above lunar limb at crescent's center, and to the arc a of solar disk visible. The latter is easily calculated in centerline geometry.

The illuminating area $\mathrm{A}$ is the small crescent on the left of fig. 3. The Sun is smaller, being total the eclipse. The Moon is superimposed. The solar height above lunar limb is $\mathrm{h}=\mathrm{Rs}-(\mathrm{Rm}-(\delta$-vt $))=(\mathrm{Rs}-\mathrm{Rm})$ $\mathrm{vt}+\delta=\mathrm{vt}$.

The difference $(\mathrm{Rm}-\mathrm{Rs})=\delta$. At the moment of totality $\mathrm{t}=0$, and $\mathrm{h}=0$. Moreover being $\cos (\beta)=-\cos (\alpha)$ the $\operatorname{arc} \alpha$ is obtained from the theorem of Carnot:

$\cos (\alpha)=\left(\mathrm{R}^{2} \mathrm{~m}-\mathrm{R}^{2} \mathrm{~s}-(\delta-\mathrm{vt})^{2}\right) /(2 \mathrm{Rs}(\delta-\mathrm{vt})) \sim 1-\mathrm{vt} / \mathrm{Rs}$

For small angles and $\mathrm{t} \rightarrow 0 \cos (\alpha) \sim 1$-vt/Rs; expanding in Taylor series the cosine we obtain $\cos (\alpha) \sim 1-\alpha^{2} / 2$ and consequently $\alpha \sim \sqrt{ }(2 \mathrm{vt} / \mathrm{Rs})$,

and the corresponding arc is $a=\alpha R s=\sqrt{ }(2 R s \cdot v \cdot t)$.

The area $A \sim h \cdot a / 2=\sqrt{ }(R s / 2) \cdot(v \cdot t)^{3} / 2 \sim t^{3} / 2$.

At shadow's limits (fig. 4) the geometry is the same, but the distance of solar and lunar centers is now $q=\sqrt{ }\left(\delta^{2}+(\mathrm{vt})^{2}\right)$ and for $\mathrm{t} \rightarrow 0 q \sim \delta\left(1+(\mathrm{vt})^{2} / 2 \delta^{2}\right)$.

The height of solar limb above the lunar one is now $\mathrm{h}=\operatorname{Rs}-(\operatorname{Rm}-q)=q-\delta \sim(\mathrm{vt})^{2} / 2 \delta \sim \mathrm{t}^{2}$. 


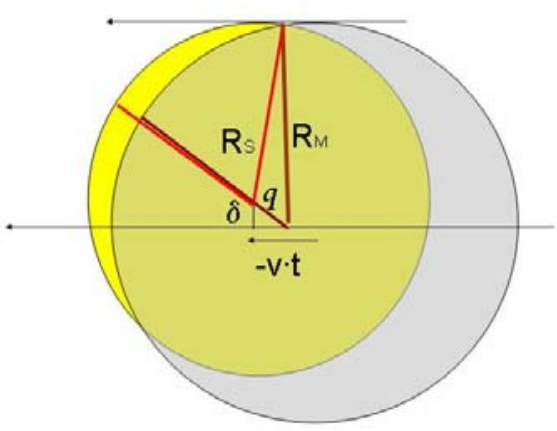

FIGURE 4. Geometry for shadow's limit case. The distance from lunar and solar centers $q$ plays the same role of $(-\mathrm{v} \cdot \mathrm{t})$ in centerline example of fig. 3 .

Because of cosine Taylor expansion the angle is now $\cos (\alpha) \sim 1-(\mathrm{v} \cdot \mathrm{t})^{2} /(2 \delta \cdot \mathrm{Rs}), \quad$ therefore $\alpha \sim \mathrm{v} \cdot \mathrm{t} / \sqrt{ }(\delta \cdot \mathrm{Rs})$, $\mathrm{a} \sim \sqrt{ }(\mathrm{Rs} / \delta) \cdot \mathrm{v} \cdot \mathrm{t}$ and consequently $\mathrm{A} \sim \sqrt{ }(\mathrm{Rs} / \delta) \cdot(\mathrm{v} \cdot \mathrm{t})^{3} / 4 \delta$.

Taylor expansion for small $t$ have been calculated for the instants around totality, and they show clearly that illumination changes differently from centerline to grazing eclipse locations. Assuming $\delta=104 \mathrm{arcsec}$, $\mathrm{Rs}=960$ arcsec and $\mathrm{v}=0.5 \mathrm{arcsec} / \mathrm{s}$ as in Egyptian eclipse on centerline sun area Ac 7.75.t3 $3 / 2 / \operatorname{arcsec}^{2}$.

On graze's limits $\mathrm{Ag} \sim 2 \cdot 10^{-3} \cdot \mathrm{t}^{3} / \mathrm{arcsec}^{2}$.

Solar corona has a magnitude of $\mathrm{M} \sim-16$, approximately 10 magnitudes fainter than the full disk. A difference of 10 magnitudes corresponds to a factor $100 \times 100=10^{4}$ in intensity, and in exposed area (neglecting solar limb darkening function).

Assuming that solar corona ceases to be visible when the exposed photospheric area equals its luminosity this area has to be Asat $=\pi \mathrm{Rs}^{2} / 10^{4}=290 \mathrm{arcsec}^{2}$.

On centerline $A c=290 \operatorname{arcsec}^{2}$ after $11.2 \mathrm{~s}$, while on shadow's limits it occurs after $52.5 \mathrm{~s}$.

With a videocamera aiming directly to the eclipsing Sun the corona has been visible for minutes on shadow's limit, and with naked eye, once covered the slowly growing luminous crescent of photosphere, corona has been visible 4 minutes after totality.

\section{Totality: Darkness Duration near Shadows Limits}

The duration of darkness, in case of total eclipses, increases with distance $d$ inside the umbral path. To the calculation made with perfectly circular Moon it is necessary to apply the corrections due to irregular lunar limbs. We used David Herald's Baily Beads program.

The maximum duration of darkness depends on the orbital velocity of the Moon (relative to the Sun), its parallactic velocity and on the difference $\delta=$ Dm-Ds between lunar and solar diameters.

$\mathrm{Tmax}=\delta /(\mathrm{Vs}-\mathrm{Vm}-\mathrm{Vp})$ vectorial sum, for the Egyptian eclipse $\mathrm{Vs}=0.04 \mathrm{arcsec} / \mathrm{s} \quad \mathrm{PA}=+23.2^{\circ} \quad \mathrm{Vm}=0.625$ $\operatorname{arcsec} / \mathrm{s} \mathrm{PA}=+28.4^{\circ}, \mathrm{Vp}=0.23 \mathrm{arcsec} / \mathrm{s} \mathrm{PA}=180^{\circ}$ and $\delta=104 \mathrm{arcsec}$, so $\mathrm{T} \max \sim 4 \mathrm{~m} 07 \mathrm{~s}$.

Drawing a circle of radius $\delta$ it is the location of all possible positions of the center of the Sun at the beginning or at the end of totality. For each observing point located at distance $\mathrm{d}<\mathrm{dmax}$ from centerline the duration of totality $\mathrm{T}(\mathrm{d})$ is proportional to the chord C(d). Similarly the distance dmax of the shadow's limits from centerline is proportional to $\delta$.

$\mathrm{d}=\delta \cdot \mathrm{d}[\mathrm{Km}] / \mathrm{dmax}[\mathrm{Km}]$

$\mathrm{d}=\delta \cdot \sin (\theta) \quad \theta=\arcsin (\mathrm{d} / \delta)$

$\mathrm{C}(\mathrm{d})=2 \cdot \delta \cdot \cos (\arcsin (\mathrm{d} / \delta))$

$\mathrm{T}(\mathrm{d})=\mathrm{Tmax} \cdot \cos (\arcsin (\mathrm{d} / \delta))$

and for $\mathrm{d} \rightarrow \delta, \mathrm{d}=\delta-\varepsilon$ with $\varepsilon \rightarrow 0$ the distance from shadows' limits. Taylor expansion with $\varepsilon \rightarrow 0$ of $\mathrm{T}(\varepsilon) \approx \operatorname{Tmax} \sqrt{ }(2 \varepsilon / \delta) \quad$ just inside the shadow's limits. The duration of totality rises as square root of distance from shadows limits. In Egyptian eclipse dmax $\sim 90 \mathrm{Km}, T \max \sim 250 \mathrm{~s}, \mathrm{~T}(1 \mathrm{~km}) \sim 37 \mathrm{~s}$, and $\mathrm{T}(100 \mathrm{~m}) \sim 12 \mathrm{~s} ; \mathrm{T}(50 \mathrm{~m}) \sim 8.4 \mathrm{~s} ; \mathrm{T}(10 \mathrm{~m}) \sim 3.8 \mathrm{~s}$.

With $\mathrm{T}(\varepsilon)$ formula it is possible to extrapolate the exact limit of the shadow with some data on totality recorded within the first $100 \mathrm{~m}$ of shadow: the experience of the employees in Manhattan eclipse.

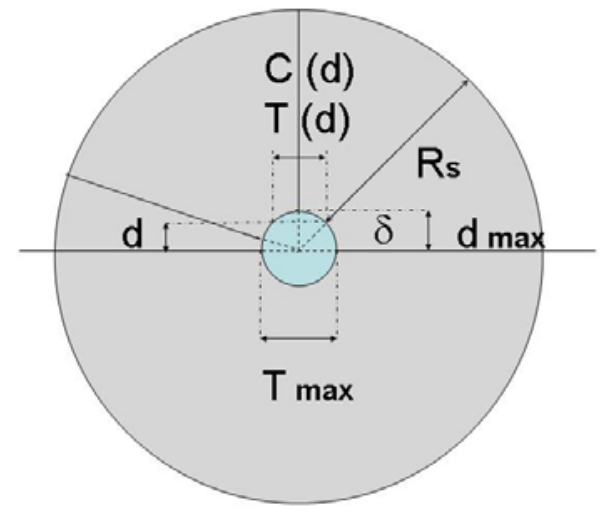

FIGURE 5. Geometry for calculation of darkness duration. The big circle is the Moon, and the smaller one represents the allowance for the solar center in order to have totality, out of this circle a part of photosphere is exposed. Rs is the solar radius, $d$ the distance from centerline, $d$ max is 
half of the shadow width. T(d) and Tmax the duration of totality at $\mathrm{d}$ and at centerline.

\section{HYBRID ECLIPSES}

In case of an hybrid eclipse (annular-total) in which the difference between centerline and shadow's limits can be limited to a few kilometers (as in the case of 1567 April, 9), the normalizations of Ac and Ag change yielding similar behaviours.

For the eclipse of Clavius $\delta \sim 6$ arcsec Rs=953.5 $\operatorname{arcsec}$ and $\mathrm{v}=0.55 \mathrm{arcsec} / \mathrm{s}$.

$\mathrm{Ag}=0.087 \cdot \mathrm{t}^{3}$ and $\mathrm{Ac}=8.91 \cdot \mathrm{t}^{3} / 2$, so that those areas become equal after $t \sim 22$ s. Solar corona, in good atmospheric conditions, was visible after and before totality for $15 \mathrm{~s}$ on shadow's limits and for $10 \mathrm{~s}$ on centerline. This fact can explain the observations reported by Clavius on his Commentarius in Sphaera (starting from 1581 edition) of a ring surrounding the Sun at maximum eclipse. With $\delta \sim 6$ arcsec the total darkness could not last more than $11 \mathrm{~s}$.

If the Clavius' eclipse was annular, the solar radius would have been 3 arcsec larger than average, a change which is not acceptable under astrophysical conditions of our star (Sofia, 2006). Clavius could observe solar corona during that hybrid eclipse, broken by several Baily beads, as claimed by Stephenson et al. (1997).

Even if Kepler asked Clavius about this eclipse, in order to discuss the nature of that ring (possible lunar atmosphere) Clavius twice answered that he saw a ring. The report of that eclipse is the first about an annular one (being actually an hybrid one) and it has been used to recover the value of the parameter $\Delta t$ in 1567, making reasonable hypotheses on the observer's location.

$\Delta \mathrm{t}=\mathrm{UT}-\mathrm{ET}$ (AT) is the difference between Universal Time and the Ephemeris Time. Universal Time is related to the meridian transit of the Sun or of a star, while Ephemeris Time to planetary orbital motions. Since the Earth spin rate changes slightly, it happened that since 1567 a meridian transit would appear in delay of about 3 minutes with respect to an atomic clock (Atomic Time, AT) running uniformly since that date.

For a total eclipse visible in Rome $\left(41.9^{\circ} \mathrm{N}\right)$ a $\Delta \mathrm{t}$ of 3 minutes implies a shift of $40 \mathrm{~km}$ of the shadow's zone. Since the eclipse of 1567 had a shadow width of $10 \mathrm{~km}$, Clavius' observation (in the former location of Collegio Romano in Casa Salviati, at the South-Eastern corner of Piazza del Collegio Romano) could allow to evaluate $\Delta \mathrm{t}$ within a few seconds.

\section{SOLAR RADIUS CORRECTIONS}

Baily Beads data consist in UTC timing of dis/appearance of a bead and its Watts (Position) angle. It happens that the system used to transport and synchronize video with UTC can experience some systematic error (watch error).

UTC also is generally different by dUT1 from UT and dUT1 is available daily down to $1 \mathrm{~ms}$ of accuracy from IERS service.

DE200 ephemerides of the Moon, used by D. Herald's program can have an uncertainty $\leq 1 \operatorname{arcsec}$ in lunar longitude, solar ephemerides have a better accuracy.

An error on UTC synchronization acts shifting the observer forward or backward with respect of the shadow (fixed), while an error on lunar longitude shifts the lunar limb backward or toward the solar one, modifying the time of bead's dis/appearance and the position of the shadow. Also a shift in lunar latitude is possible.

In order to distinguish the contributions of lunar longitude, latitude, solar longitude, lunar parallax and UTC synchronization the residuals hi=Si-Mi SolarLunar limbs from ephemerides with average solar diameter, represent the excess or defect with respect to 959.63 arcsec at unit distance (1AU). The average $<$ hi $>$ is the correction to the average radius, and its standard deviation $\sigma$ is the statistical uncertainty on that correction.

In case of Saros-connected eclipses the beads are the same, and each one contributes in the same way to $\sigma$, therefore if the uncertainty on timing is minimal the radius corrections differences are the effective solar radius variation. The same consideration is repeated about polar beads (Dunham, 2005) from one eclipse to another.

The kinematics of the eclipse is given by the vectorial equation

$$
\overrightarrow{\mathrm{V}}_{\text {tot }}=\overrightarrow{\mathrm{V}}_{\text {Moon }}+\overrightarrow{\mathrm{V}}_{\text {parM }}-\overrightarrow{\mathrm{V}}_{\text {Sun }}
$$

For each eclipse the three components are different and calculated from ephemeredes of Sun and Moon. 
Three kind of corrections to data are made:

$\Delta \mathbf{t}$ timing, hereinafter $\Delta \mathrm{t}$, it is applied to the whole velocity $\mathrm{V}$ tot, to consider dUT1 corrections to UTC and systematic shift of local watch.

$\Delta \mathbf{t}$ moon is applied only to $\mathrm{V}$ Moon, to consider corrections to lunar longitude, and a correction to lunar latitude $\Delta$ Lat_moon is applied perpendicularly to $\mathrm{V}$ Moon. In the Egyptian total eclipse of March 29,2006 these corrections $(\Delta \mathrm{t}=-0.5 \mathrm{~s}, \Delta \mathrm{t}$ moon $=$ $0.23 \mathrm{~s}=0.14$ arcsec; $\Delta$ Lat_moon $=0.13$ arcsec) minimized the standard deviation of residuals hi $=\mathrm{Si}$ Mi, where Mi is the Watts' profile for each Position Angle (i), corrected according to Morrison and Appleby (1981). As evident from figure 6 the beads at Southern limit, where we observed the eclipse, does not allow to discriminate between a correction $\pm \Delta \mathrm{R}$ of solar radius and a $\pm \Delta$ Lat_moon, because of lack of data in the $270^{\circ}-360^{\circ}$ range of position angles (Northern limb observation).

Therefore the uncertainty on $\Delta \mathrm{R}=-0.38$ arcsec is 0.30 arcsec. In comparison the annular eclipse of 2006 September, 22 in French Guyana (fig. 7) required corrections $\Delta \mathrm{t}=-0.01 \mathrm{~s}, \Delta \mathrm{t} \_$moon $=-1.29 \mathrm{~s}=-0.63 \operatorname{arcsec}$ and $\Delta$ Lat_moon $=0.11$ arcsec. With a final $\Delta \mathrm{R}=-0.01$ $\operatorname{arcsec} \pm 0.17$ arcsec.

On the lunar longitude corrections the amount is -0.14 " in March and -0.63" in September, reasonable for lunar DE200 ephemerides, as well as the lunar latitude shifts.

The $-0.5 \mathrm{~s}$ watch error on March was due to Garmin II+ GPS display delay of that amount.

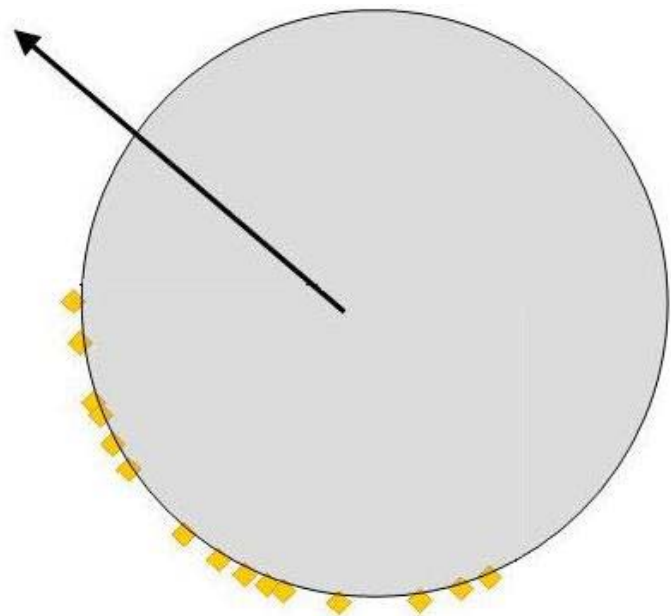

FIGURE 6. Position angles of Baily Beads used in data analysis of Egyptian total eclipse. North is up, angles are counterclockwise. They range from $90^{\circ}$ to $200^{\circ}$. The relative velocity vector of the Moon with respect to the Sun after inclusion of parallax effect is drawn as an arrow.

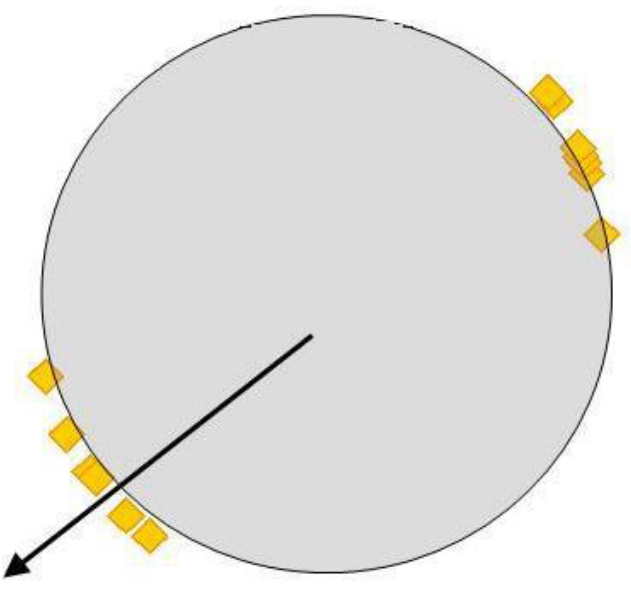

FIGURE 7. Position angles of Baily Beads used in data analysis of French Guyana annular eclipse. North is up, angles are counterclockwise. They are grouped around $130^{\circ}\left(3^{\text {rd }}\right.$ contact, end of annularity) and $290^{\circ}\left(2^{\text {nd }}\right.$ contact, beginning of annularity). The arrow is lunar relative velocity to the Sun.

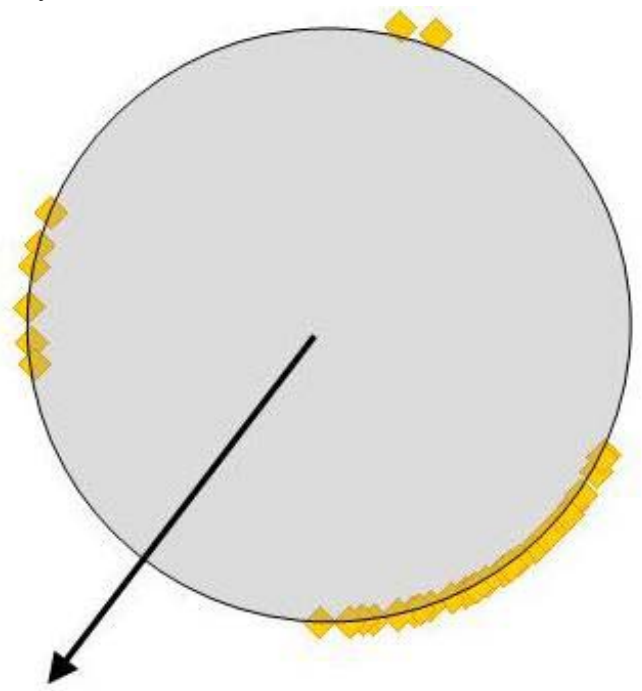

FIGURE 8. Position angles of Baily Beads used in data analysis of Spain annular eclipse, Oct. $3^{\text {rd }}, 2005$. North is up, angles are counterclockwise. Dr. W. Strickling at N limit (left and top beads), and C. Perelli, S limit (bottom and right beads) sampled fairly well all Position angles.

In the annular eclipse of Spain (October, $3^{\text {rd }} 2005$ ) we obtained data from 2 observers at both limbs and the corrections are $\Delta \mathrm{t}=-0.79 \mathrm{~s}$ for the $\mathrm{N}$ limit observations and $\Delta \mathrm{t}=0.09 \mathrm{~s}$ for $\mathrm{S}$. $\Delta \mathrm{t}$ moon $=0.14 \mathrm{~s}$ and $\Delta$ Lat_moon $=0.0$ arcsec. With a final $\Delta \mathrm{R}=0.00$ $\operatorname{arcsec} \pm 0.17$ arcsec. 


\section{CONCLUSIONS}

The data on the Egyptian eclipse, with only beads from Southern limit does not allow to recover the correction $\Delta \mathrm{R}$ to average solar radius at unit distance. There exist a single observation within the Northern limit of a $30 \mathrm{~s}$ of darkness, with uncertain observer's position data. The situation is better for Spanish and Guyanese annular eclipses, because there are beads well sampled covering more than $180^{\circ}$.

From October 3, 2005 (S) to September 22, 2006 (G) there is no appreciable change in solar radius.

$\Delta R(S)=0.00 \pm 0.17$ and $\Delta R(G)=-0.01 \pm 0.17$ arcsec.

Since the Sun is in its minimum activity that could be its maximum radius according to Haberreiter et al. (2005). If solar radius correction is $\Delta \mathrm{R}=0$ at its maximum it means that the Sun is shrinking during the last decades. The effect of limb darkening (Rogerson, 1959) combined with atmospheric and optical filtering produce an artificial reduction of the observed photospheric radius (Sigismondi and Oliva, 2005b) but that effect seems to be negligible at this level of accuracy.

\section{ACKONWLEDGMENTS}

Thanks to David Dunham and Wayne Warren who introduced me in to this field, and see caption of fig. 1 for other people with whom I am indebted. Special thanks to Prof. Alessandro Cacciani, who welcomed my studies in his lab. He deceased on July 9, 2007.

\section{REFERENCES}

1. Brown E.W., Discussion of observations of the moon at and near the eclipse of 1925 January 24, Astronomical Journal 37, 9-19 (1926).

2. Brown T. M., Christensen-Dalsgaard J., Accurate Determination of the Solar Photospheric Radius, Astrophysical J. 500, L195-L198 (1998).

3. Clavius, C., Commentarius in Sphaeram, Venezia $\left(1581^{2}\right)$

4. Damé, L. et al, PICARD: simultaneous measurements of the solar diameter, differential rotation, solar constant and their variations, Advances in Space Research 24, 205-214 (1999).

5. Dunham D. W., Dunham J.B., Observing Total Solar Eclipses from Near the Edge of the Predicted Path, Lunar Dynamics and Observational Coordinate Systems. Moon 8, 1973. Revised abstracts published as Lunar Sci. Inst. Contrib. 135. Edited by $M$. Moutsoulas, 546 (1973).

6. Dunham D.W., Sofia S., Fiala A.D., Muller P.M., Herald, D., Observations of a probable change in the solar radius between 1715 and 1979, Science 210, 1243-1245 (1980).
7. Dunham D. W. et al., Accuracy of Solar Radius Determinations from Solar Eclipse Observations, and Comparison with SOHO Data, 2005 SORCE Science Meeting September 14-16, Durango, Colorado. (2005).

8. Eddy, J.A. and A. A. Boornazian, Bull. Am. Astron. Soc. 11, 437 (1979).

9. Egidi, A., B. Caccin et al., High-Precision Measurements of the Solar Diameter and Oblateness by the Solar Disk Sextant (SDS) Experiment, Solar Physics 235, 407-418 (2006).

10. Endal A. S., S. Sofia and L. W. Twigg, Changing in Solar Luminosity and Radius Following Secular Perturbation in the Convective Envelope, Astrophysical Journal 294, 748-757 (1985).

11. Fiala, A.D.; Dunham, D. W.; Sofia, S., Variation of the Solar Diameter from Solar Eclipse Observations, 1715-1991, Solar Physics 152, 97-104 (1994).

12. Halley, E., Observation of Late Total Solar Eclipse on the 22nd, April [...], Philosophical Transactions of the R. Soc. of London XXIX 245-262 (1714-1716).

13. Herald, D., WinOccult 3.1.0-3.6 http://www.lunaroccultations.com/iota/occult3.htm (2001-2006)

14. Maffei, P. La Cometa di Halley, Mondadori, Milano (1984).

15. Morrison L.V., Appleby G.M., Analysis of Lunar Occultations. MNRAS 196, 1013-1020 (1981).

16. Parkinson, J.H.; Morrison, L.V.; Stephenson, F.R., The constancy of the Solar diameter over the past 250 years, Nature 288 548-551 (1988).

17. Newton, R. R., Medieval Chronicles and Earth Rotation History, Johns Hopkins University Press (1972)

18. Povenmire, H., Graze Observers Handbook, JSB Enterprises, Indian Harbour Beach FL (1975).

19. Rogerson, B.D., The Solar Limb Intensity Profile, Astrophysical Journal 130, 985 (1959).

20. Sigismondi C., Oliva P., Solar Oblateness from Archimedes to Dicke, Nuovo Cimento B 120, 1181 (2005).

21. Sigismondi C., Oliva P., Astrometry and Relativity II: Solar Grazes \& Solar Diameter, Journal of Korean Physics Society 2006 vol.49 (S02) pp. 840-5 (2005b).

22. Sofia S., Okeefe J., Lesh J. R., Endal A.S., Solar constant - Constraints on possible variations derived from solar diameter measurements, Science 204, 1306 (1979).

23. Sofia S., Dunham D. W., Dunham J. B., Fiala A. D., Solar radius change between 1925 and 1979, Nature 304, 522-526 (1983).

24. Stephenson, F. R., J. E. Jones, and L.V. Morrison, The solar eclipse observed by Clavius in A.D. 1567, Astronomy and Astrophysics 322, 347-351 (1997).

25. Stephenson F.R., Historical Eclipses and Earth's Rotation, Cambridge University Press (1997).

26. Thuillier, G., S. Sofia and M. Haberreiter, Past, Present and Future Measurements of the Solar Diameter, Advances in Space Research 35 329-340 (2005).

27. Watts, C.B., The Marginal Zone of the Moon, Astronomical Papers prepared for the use of the American Ephemeris and Nautical Almanac (United States Government Printing Office, Washington XVII (1963). 